\title{
A Weak Form of the Conjugate Gradient FFT Method for Two-Dimensional TE Scattering Problems
}

\author{
Peter Zwamborn and Peter M. van den Berg
}

\begin{abstract}
The problem of two-dimensional scattering of a transversal electric (TE) polarized wave by a dielectric object can be formulated in terms of a hypersingular integral equation, in which a grad-div operator acts on a vector potential. The vector potential is a spatial convolution of the free-space Green's function and the contrast source over the domain of interest. A weak form of the integral equation for the unknown electric flux density is obtained by testing it with rooftop functions. As the next step, the vector potential is expanded in a sequence of the rooftop functions and the grad-div operator is integrated analytically over the dielectric object domain only. This method shows excellent numerical performance.
\end{abstract}

\section{INTRODUCTION}

$\mathbf{I}^{\mathrm{N}}$ $\mathrm{N}$ the development of biological applications in electromagnetics, numerical schemes are needed for fast and accurate modeling of the electromagnetic field inside a strongly inhomogeneous and lossy dielectric object exposed to a known incident field. The problem of the electromagnetic scattering by an inhomogeneous dielectric object can be formulated in terms of an integral equation for the electric field over the domain of the object. Numerous methods have been developed, and it is not our objective to survey them all. Instead, we concentrate on the $k$-space methods. It is our opinion that methods of this type are applicable for three-dimensional electromagnetic scattering problems owing to their storage and computational efficiency.

The first method for solving the electric field integral equation over the domain of a dielectric object was developed by Richmond [1], [2]. Here the method of moments was used with pulse expansion functions and point matching. The method of moments, however, needs an inversion of a (large) matrix, limiting the application of this method. Bojarski [3] introduced the $k$-space method, obtaining an iterative approach that reduces the storage and

Manuscript received August 6, 1990; revised December 4, 1990. This work was supported by a NATO Grant $(0230 / 88)$ and by research grants from the Stichting Fund for Science, Technology and Research (a companion organization to the Schlumberger Foundation in the U.S.), from Schlumberger-Doll Research, Ridgefield, CT, and from Etudes et Productions Schlumberger, Clamart, France.

The authors are with the Department of Electrical Engineering, Delft University of Technology, P.O. Box 5031, 2600 GA Delft, The Netherlands.

IEEE Log Number 9144268. computation by using a fast Fourier transform algorithm for computation of the spatial convolution that occurs in the integral equation. Subsequently, the conjugate gradient method combined with the fast Fourier transform was developed [4], [5]. For the three-dimensional problems and the two-dimensional case of TE polarization, there were serious doubts as to the applicability of this conjugate gradient FFT method using pulse expansion functions [6], [7]. Borup et al. [8] showed that in the conjugate gradient FFT method serious inaccuracies are observed for cylindrical objects in the TE polarization case. Recently, Joachimowicz and Pichot [10] analyzed the source of these errors. They introduced an integral equation formulation using generalized functions and included surface integrals in relation to surface charges at the discontinuity surfaces. Better numerical performance has been demonstrated, although erroneous behavior can still be observed.

Recently, the weak form of the conjugate gradient FFT method has proved to be an efficient and accurate scheme for solving perfectly conducting plate scattering problems [9]. Therefore, in the present paper, we employ this weak form of the integral equation by testing the integral equation with rooftop functions. Subsequently, a suitable expansion procedure of the vector potential in the integral equation is carried out. The first result is that the grad-div operator acting on the vector potential of this hypersingular integral equation is integrated analytically over the domain of the dielectric object only. The second result is that we have maintained the simple scalar form of the convolution structure of the vector potential (in fact, two scalar convolutions). This means that the computation time of each iteration of our present scheme is even less than the one of all previous conjugate gradient FFT methods described above. Finally, it is noted that we formulate the integral equation in terms of the unknown electric flux density rather than in terms of the electric field. The enforced continuity of the electric flux density yields a correct implementation of the boundary condition at the interfaces of (strong) discontinuity. Therefore, the present scheme is much simpler than the one of Joachimowizc and Pichot [10]. No surface integrals that are directly related to surface charges have to be introduced. 
We present numerical results for some two-dimensional test problems. First, these two-dimensional test problems are less computationally involved. Second, the two-dimensional TE case exhibits stronger polarization effects than the three-dimensional case. Effects of strong inhomogeneities are more visible in the two-dimensional TE case than in the three-dimensional case. Numerical computations have been carried out for coaxially layered cylinders. The numerical results are compared with existing analytical solutions, and it is demonstrated that the present weak formulation shows excellent results. Comparing the results with the ones presented by Joachimowicz and Pichot [10], it is directly observed that the weak form of the conjugate gradient FFT method produces much more accurate numerical results. This indicates that the present weak formulation of the conjugate gradient FFT method can be considered a simple and efficient tool for solving (strongly) inhomogeneous lossy dielectric scattering problems.

\section{The Domain Integral Equation}

The vectorial position in the two-dimensional space is denoted by $\boldsymbol{x}=\left(x_{1}, x_{2}\right)$. The unit vectors in the $x_{1}$ and $x_{2}$ directions are given by $i_{1}$ and $i_{2}$. The time factor $\exp (-i \omega t)$ has been used for the field quantities in the frequency domain. We consider the problem of scattering by an inhomogeneous dielectric object with complex permittivity

$$
\epsilon(\boldsymbol{x})=\epsilon_{r}(\boldsymbol{x}) \epsilon_{0}+i \frac{\sigma(\boldsymbol{x})}{\omega}
$$

where $\epsilon_{r}$ denotes the relative permittivity of the object with respect to the lossless and homogeneous embedding with permittivity $\epsilon_{0}$, and $\sigma$ denotes the electric conductivity of the object. The incident electric field is denoted by $\boldsymbol{E}^{i}=\left(E_{1}^{i}, E_{2}^{i}\right)$. In this paper, we formulate the scattering problem as a domain integral equation for the unknown electric flux density $\boldsymbol{D}=\left(D_{1}, D_{2}\right)$ over the object domain $S$ as

$$
E^{i}(x)=\frac{D(x)}{\epsilon(x)}-\left(k_{0}^{2}+\operatorname{grad} \operatorname{div}\right) A(x), \quad x \in S .
$$

where $k_{0}=\omega\left(\epsilon_{0} \mu_{0}\right)^{1 / 2}$ and the vector potential $A=$ $\left(A_{1}, A_{2}\right)$ is given by

$$
A(x)=\mathscr{F}^{-1}\left[\mathscr{F}[G(x)] \mathscr{F}\left[\chi(x) \frac{D(x)}{\epsilon_{0}}\right]\right]
$$

in which $\mathscr{F}$ and $\mathscr{F}^{-1}$ denote the forward and inverse two-dimensional spatial Fourier transforms, and the normalized contrast function, $\chi$, is given by

$$
\chi(x)=\frac{\epsilon(x)-\epsilon_{0}}{\epsilon(x)} .
$$

Further, the two-dimensional Green's function, $G$, is given by

$$
G(x)=\frac{i}{4} H_{0}^{(1)}\left(k_{0}|x|\right), \quad x \in \mathbb{R}^{2} .
$$

\section{Testing and Expansion Procedure}

We first introduce a discretization in the spatial domain $\boldsymbol{x}=\left(x_{1}, x_{2}\right)$. We use a square mesh with a grid width of $\Delta x$ in both directions. For convenience, the discrete values of $\boldsymbol{x}$ are given by a staggered grid [9]:

$$
\begin{aligned}
& x_{n, m}^{(1)}=\left(\left(n-\frac{1}{2}\right) \Delta x, m \Delta x\right) \\
& \boldsymbol{x}_{n, m}^{(2)}=\left(n \Delta x,\left(m-\frac{1}{2}\right) \Delta x\right) .
\end{aligned}
$$

The boundary of the discretized object now consists of straight line segments parallel to the $x_{1}$ or $x_{2}$ axis. We assume that the discretized boundary $\partial S$ of the scattering domain $S$ lies completely in the embedding where $\chi=0$. This is always possible, since we can extend the definition of the scattering domain $S$ by extending it with a zero contrast function $\chi$. In each square subdomain with center $x=(n \Delta x, m \Delta x)$ and dimensions $\Delta x \times \Delta x$, we assume the complex permittivity to be constant with values $\epsilon_{n, m}$. Note that jumps in the (complex) permittivity function may occur at $x_{1}=(n-1 / 2) \Delta x$ and at $x_{2}=(m-$ 1/2) $\Delta x$.

In order to cope with the grad-div operator in (2), we test the two scalar equations of (2). Therefore we multiply both sides of this equation by a vectorial testing function $\boldsymbol{\psi}^{(p)}\left(\boldsymbol{x}_{n, m}^{(p)}-\boldsymbol{x}\right), p=1,2$, and integrate with respect to $\boldsymbol{x}$ over the object domain $S$. The testing vector function $\boldsymbol{\psi}^{(p)}(\boldsymbol{x})=\psi^{(p)}(\boldsymbol{x}) i_{p}$ is a suitably chosen vector function that will be defined later. We then obtain

$$
\begin{aligned}
\int_{S} \psi^{(p)}\left(x_{n, m}^{(p)}-x\right) E_{p}^{i}(x) d x \\
=\int_{S} \psi^{(p)}\left(x_{n, m}^{(p)}-\boldsymbol{x}\right) \frac{D_{p}(\boldsymbol{x})}{\epsilon(\boldsymbol{x})} d \boldsymbol{x} \\
\quad-k_{0}^{2} \int_{S} \psi^{(p)}\left(\boldsymbol{x}_{n, m}^{(p)}-\boldsymbol{x}\right) A_{p}(\boldsymbol{x}) d \boldsymbol{x} \\
+\int_{S} \partial_{p} \psi^{(p)}\left(\boldsymbol{x}_{n, m}^{(p)}-\boldsymbol{x}\right) \operatorname{div} \boldsymbol{A}(\boldsymbol{x}) d \boldsymbol{x} \\
\quad \boldsymbol{x}_{n, m}^{(p)} \in S, \quad p=1,2 .
\end{aligned}
$$

The last integral on the right-hand side of (7) is obtained by using Gauss's theorem on each subdomain where $\psi^{(p)}\left(\boldsymbol{x}_{n, m}^{(p)}-\boldsymbol{x}\right) \operatorname{div} \boldsymbol{A}(\boldsymbol{x})$ is continuously differentiable and by using the continuity of the normal component of this function through the interfaces between these subdomains. In the derivation of (7) we have assumed that $\psi^{(p)}\left(x_{n, m}^{(p)}-\boldsymbol{x}\right)$ vanishes for $\boldsymbol{x} \in \partial S$. Further, $\partial_{p}$ denotes the partial derivative with respect to $x_{p}, p=1,2$. In (7) we expand the electric flux density, $D$, and the vector potential, $A$, as

$$
\begin{aligned}
& D_{p}(\boldsymbol{x})=\epsilon_{0} \sum_{k, l} d_{p ; k, l} \psi^{(p)}\left(\boldsymbol{x}-\boldsymbol{x}_{k, l}^{(p)}\right) \\
& A_{p}(\boldsymbol{x})=\sum_{k, l} a_{p ; k, l} \psi^{(p)}\left(\boldsymbol{x}-\boldsymbol{x}_{k, l}^{(p)}\right) .
\end{aligned}
$$


Using these expansions in the right-hand side of (7), carrying out the divergence operator, and interchanging the order of integration and summation, we obtain the weak form of the integral equation

$$
\begin{aligned}
& e_{p}^{i}\left(\boldsymbol{x}_{n, m}^{(p)}\right)= \sum_{k, l}\left[u^{(p)}\left(\boldsymbol{x}_{n, m}^{(p)} ; \boldsymbol{x}_{k, l}^{(p)}\right) d_{p ; k, l}\right. \\
&-k_{0}^{2} v^{(p)}\left(\boldsymbol{x}_{n, m}^{(p)} ; \boldsymbol{x}_{k, l}^{(p)}\right) a_{p ; k, l} \\
&\left.+\sum_{q=1}^{2} w_{p, q}\left(\boldsymbol{x}_{n, m}^{(p)} ; \boldsymbol{x}_{k, l}^{(q)}\right) a_{q ; k, l}\right], \\
& \boldsymbol{x}_{n, m}^{(p)} \in S, \quad p=1,2
\end{aligned}
$$

in which

$$
\begin{aligned}
e_{p}^{i}\left(\boldsymbol{x}_{n, m}^{(p)}\right)= & \int_{S} \psi^{(p)}\left(\boldsymbol{x}_{n, m}^{(p)}-\boldsymbol{x}\right) E_{p}^{i}(\boldsymbol{x}) d \boldsymbol{x} \\
u^{(p)}\left(\boldsymbol{x}_{n, m}^{(p)} ; \boldsymbol{x}_{k, l}^{(p)}\right)= & \int_{S} \psi^{(p)}\left(\boldsymbol{x}_{n, m}^{(p)}-\boldsymbol{x}\right) \\
& \cdot \frac{\epsilon_{0}}{\epsilon(\boldsymbol{x})} \psi^{(p)}\left(\boldsymbol{x}-\boldsymbol{x}_{k, l}^{(p)}\right) d \boldsymbol{x} \\
v^{(p)}\left(\boldsymbol{x}_{n, m}^{(p)} ; \boldsymbol{x}_{k, l}^{(p)}\right)= & \int_{\boldsymbol{S}} \psi^{(p)}\left(\boldsymbol{x}_{n, m}^{(p)}-\boldsymbol{x}\right) \psi^{(p)}\left(\boldsymbol{x}-\boldsymbol{x}_{k, l}^{(p)}\right) d \boldsymbol{x} \\
w_{p, q}\left(\boldsymbol{x}_{n m}^{(p)} ; \boldsymbol{x}_{k, l}^{(q)}\right)= & \int_{S} \partial_{p} \psi^{(p)}\left(\boldsymbol{x}_{n, m}^{(p)}-\boldsymbol{x}\right) \partial_{q} \psi^{(q)}\left(\boldsymbol{x}-\boldsymbol{x}_{k, l}^{(q)}\right) d \boldsymbol{x} .
\end{aligned}
$$

In view of the partial derivatives in (14), the derivative with respect to $x_{p}$ of the function $\psi^{(p)}(x)$ must exist. Therefore we take the rooftop functions [9] as testing and expansion functions, viz.

$$
\begin{aligned}
& \psi^{(1)}(\boldsymbol{x})=\Lambda\left(x_{1} ; 2 \Delta x\right) \Pi\left(x_{2} ; \Delta x\right) \\
& \psi^{(2)}(\boldsymbol{x})=\Pi\left(x_{1} ; \Delta x\right) \Lambda\left(x_{2} ; 2 \Delta x\right)
\end{aligned}
$$

in which $\Lambda(x ; 2 \Delta x)$ is the one-dimensional triangle function with support $2 \Delta x$ in the $x$ direction, and $\Pi(x ; \Delta x)$ is the one-dimensional pulse function with support $\Delta x$ in the $x$ direction. The value of $e_{p}^{i}$ in (11) can be integrated analytically for some particular choice of $E_{p}^{i}$. With (15), we are also able to calculate the functions $u^{(p)}(\boldsymbol{x}), v^{(p)}(\boldsymbol{x})$, and $w_{p, q}(\boldsymbol{x})$ analytically. Substitution of the results in (10) leads to

$$
\begin{aligned}
e_{1}^{i}\left(\boldsymbol{x}_{n, m}^{(1)}\right)= & \sum_{k=1}^{3}\left(b_{k ; n, m}^{(1)} d_{1 ; n+k-2, m}+c_{k} a_{1 ; n+k-2, m}\right) \\
& -\sum_{k=1}^{2} \sum_{l=1}^{2} t_{k l} a_{2 ; n+k-2, m+l-1} \\
e_{2}^{i}\left(\boldsymbol{x}_{n, m}^{(2)}\right)= & \sum_{l=1}^{3}\left(b_{l ; n, m}^{(2)} d_{2 ; n, m+l-2}+c_{l} a_{2 ; n, m+l-2}\right) \\
& -\sum_{k=1}^{2} \sum_{l=1}^{2} t_{k l} a_{1 ; n+k-1, m+l-2}
\end{aligned}
$$

in which $\boldsymbol{x}_{n, m}^{(p)} \in S$. The coefficients $b_{k ; n, m}^{(p)}$ and $c_{k}$ of the vectors $\boldsymbol{b}_{n, m}^{(p)}$ and $\boldsymbol{c}$ are obtained as

$$
\begin{gathered}
b_{n, m}^{(1)}=\frac{(\Delta x)^{2}}{6}\left(\begin{array}{c}
\frac{\epsilon_{0}}{\epsilon_{n-1, m}} \\
\frac{2 \epsilon_{0}}{\epsilon_{n-1, m}}+\frac{2 \epsilon_{0}}{\epsilon_{n, m}} \\
\frac{\epsilon_{0}}{\epsilon_{n, m}}
\end{array}\right) \\
b_{n, m}^{(2)}=\frac{(\Delta x)^{2}}{6}\left(\begin{array}{c}
\frac{\epsilon_{0}}{\epsilon_{n, m-1}} \\
\frac{2 \epsilon_{0}}{\epsilon_{n, m-1}}+\frac{2 \epsilon_{0}}{\epsilon_{n, m}} \\
\frac{\epsilon_{0}}{\epsilon_{n, m}}
\end{array}\right) \\
c=-\frac{k_{0}^{2}(\Delta x)^{2}}{6}\left(\begin{array}{l}
1 \\
4 \\
1
\end{array}\right)+\left(\begin{array}{r}
-1 \\
2 \\
-1
\end{array}\right)
\end{gathered}
$$

while the coefficients $t_{k l}$ of the matrix $t$ follows from

$$
\boldsymbol{t}=\left(\begin{array}{rr}
1 & -1 \\
-1 & 1
\end{array}\right)
$$

With our particular choice of expansion functions, the quantities $d_{p ; n, m}$ and $a_{p ; n, m}$ are

$$
\begin{array}{ll}
d_{p ; n, m}=\frac{D_{p}\left(\boldsymbol{x}_{n, m}^{(p)}\right)}{\epsilon_{0}}, & p=1,2 \\
a_{p ; n, m}=A_{p}\left(\boldsymbol{x}_{n, m}^{(p)}\right), & p=1,2
\end{array}
$$

and are related via (3), in which the Fourier transforms are replaced by discrete Fourier transforms (DFT's). Substituting (8) into (3) and taking into account that the DFT is the numerical counterpart of the continuous Fourier transform when a trapezoidal integration rule is used, we arrive at

$$
\begin{array}{r}
a_{p ; n, m}=\mathrm{DFT}^{-1}\left[\mathscr{F}[G] \mathrm{DFT}\left[\chi_{n, m}^{(p)} d_{p ; n, m}\right]\right], \\
p=1,2
\end{array}
$$

in which

$$
\begin{aligned}
\chi_{n, m}^{(1)} & =\frac{\chi\left(\boldsymbol{x}_{n-1 / 2, m}^{(1)}\right)+\chi\left(\boldsymbol{x}_{n+1 / 2, m}^{(1)}\right)}{2} \\
\chi_{n, m}^{(2)} & =\frac{\chi\left(\boldsymbol{x}_{n, m-1 / 2}^{(2)}\right)+\chi\left(\boldsymbol{x}_{n, m+1 / 2}^{(2)}\right)}{2} .
\end{aligned}
$$

As next step, in (24) we have to replace the continuous transform of the Green's function, $G$, by a discrete one. In order to cope with the singularity at $\boldsymbol{x}=0$, we use a global representation consistent with our weak formulation. We integrate the Green's function over circular patches with centers at the points $\boldsymbol{x}=(n \Delta x, m \Delta x)$ and radius of $1 / 2 \Delta x$ and divide the results by the surface 

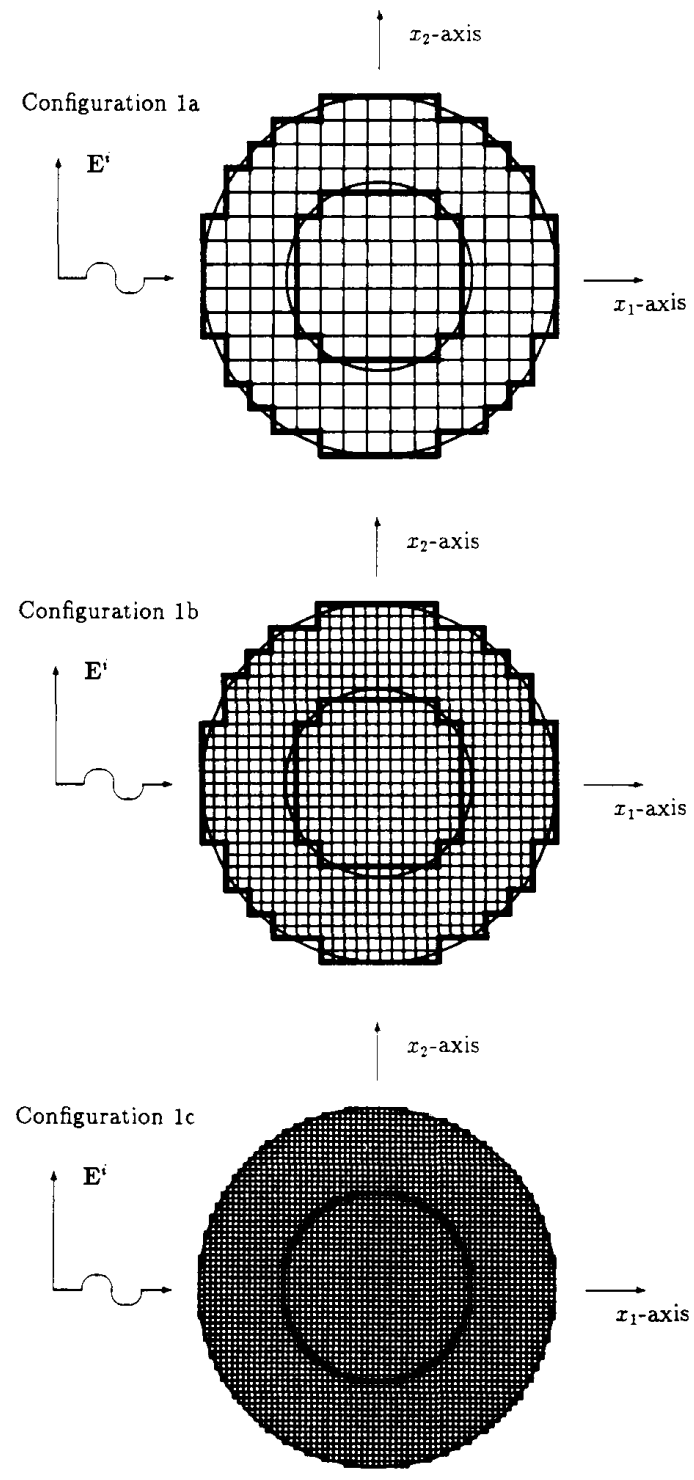

Fig. 1. The different geometries of the coaxially layered cylinder of the first test case.
Equations (16), (17), and (28) represent an operator equation for the unknown values of $d_{p ; n . m}$. To solve this operator equation iteratively, a conjugate gradient scheme [4] can now be applied.

\section{NuMERICAL IMPLEMENTATION}

Using numerical techniques yields bounded domains for the evaluation of the discrete Fourier transform. In order to obtain reliable numerical results, we have to investigate this truncation procedure [10], [11]. Using the discrete Fourier transform and the convolution theorem, the numerically approximated vector potential can be written as follows:

$$
a_{p ; n, m}=(\Delta x)^{2} \sum_{n^{\prime}, m^{\prime}} g_{n-n^{\prime}, m-m^{\prime}} j_{p: n^{\prime}, m^{\prime}}, \quad p=1,2
$$

in which the discrete functions $j_{p, n, m}$ are given by

$$
j_{p ; n, m}=\chi_{n, m}^{(p)} d_{p ; n, m} \text {. }
$$

From (30) and (4) it is easily verified that $j_{p ; n, m}=0$, $\boldsymbol{x}_{n, m}^{(p)} \notin S$. This restricts the necessary upper and lower bounds in the summation of (29). Let us assume that the domain $S$ of the object lies completely inside a rectangular domain:

$$
\begin{gathered}
\left(n_{\min }-1\right) \Delta x<x_{1}<\left(n_{\max }+1\right) \Delta x \\
\left(m_{\min }-1\right) \Delta x<x_{2}<\left(m_{\max }+1\right) \Delta x .
\end{gathered}
$$

In this rectangular domain we have $N_{S}=n_{\max }-n_{\min }+1$ mesh points in the $x_{1}$ direction and $M_{S}=m_{\max }-m_{\min }+1$ mesh points in the $x_{2}$ direction. For a correct evaluation of $a_{p ; n, m}$, if $x_{n, m}^{(p)} \in S$, it has been shown by Brigham [13] that (29) can be computed as a cyclic convolution using DFT. The relevant DFT's are defined on an extended rectangular domain with $N_{C}$ mesh points in the $x_{1}$ direction and $M_{C}$ mesh points in the $x_{2}$ direction, such that

$$
N_{C} \geqslant 2 N_{S} \quad M_{C} \geqslant 2 M_{S} .
$$

area $\pi(1 / 2 \Delta x)^{2}$. The results are [1]

$$
g_{n, m}=\frac{4}{\pi(\Delta x)^{2}} \int_{\text {patch }_{n, m}} \frac{i}{4} H_{0}^{(1)}\left(k_{0}|\boldsymbol{x}|\right) d \boldsymbol{x}=\left\{\begin{array}{l}
\left(\frac{i}{\Delta x k_{0}}\right) J_{1}\left(k_{0} \frac{\Delta x}{2}\right) H_{0}^{(1)}\left(k_{0} \sqrt{n^{2}+m^{2}} \Delta x\right) \\
\left(\frac{i}{\Delta x k_{0}}\right)\left(H_{1}^{(1)}\left(k_{0} \frac{\Delta x}{2}\right)+\frac{4 i}{\pi \Delta x k_{0}}\right) \quad \text { if } n=m=0 .
\end{array}\right.
$$

With this global representation of the Green's function, the discrete values of the vector potential (cf. (24)) are obtained as

$$
\begin{array}{r}
a_{p ; n, m}=(\Delta x)^{2} \mathrm{DFT}^{-1}\left[\operatorname{DFT}\left[g_{n, m}\right] \mathrm{DFT}\left[\chi_{n, m}^{(p)} d_{p ; n, m}\right]\right], \\
p=1,2 .
\end{array}
$$

Then, the values of $a_{p: n, m}$ are obtained as

$$
\begin{aligned}
a_{p ; n, m}=(\Delta x)^{2} \mathrm{DFT}_{N_{C}, M_{C}}^{-1}\left[\tilde{g}_{k, l} \tilde{j}_{p ; k, l}\right], \\
\boldsymbol{x}_{n, m}^{(p)} \in S, \quad p=1,2
\end{aligned}
$$




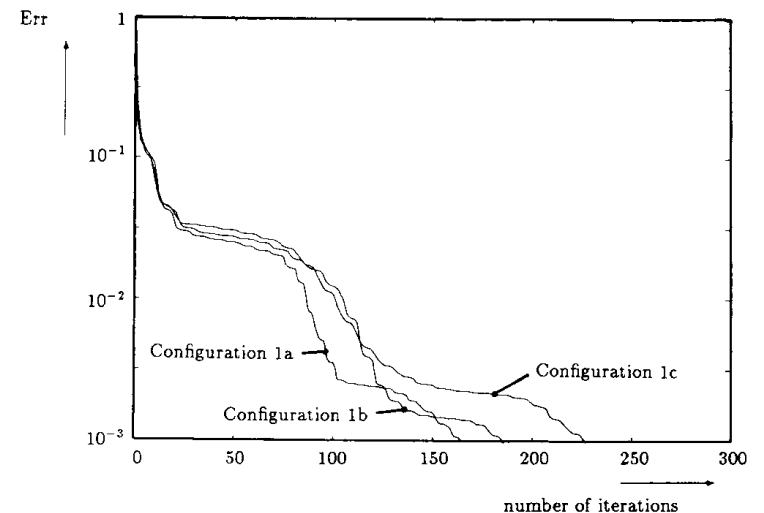

Fig. 2. Numerical convergence rate for the different geometries of the first test case.
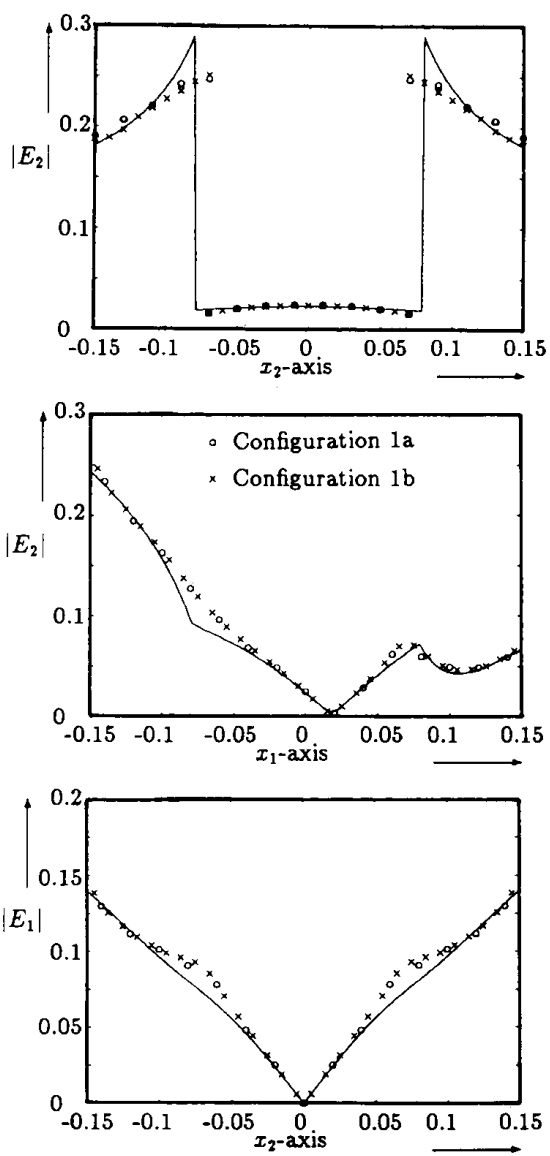

Fig. 3. Comparison between the analytic solution and the present method for the first test case.

with

$$
\begin{aligned}
\tilde{g}_{k, l} & =\operatorname{DFT}_{N_{C}, M_{C}}\left[g_{n, m}\right] \\
\tilde{j}_{p ; k, l} & =\operatorname{DFT}_{N_{C}, M_{C}}\left[j_{p ; n, m}\right] .
\end{aligned}
$$

Here, the subscripts $N_{C}$ and $M_{C}$ denote the relevant DFT numbers. If the discretization of the object is chosen
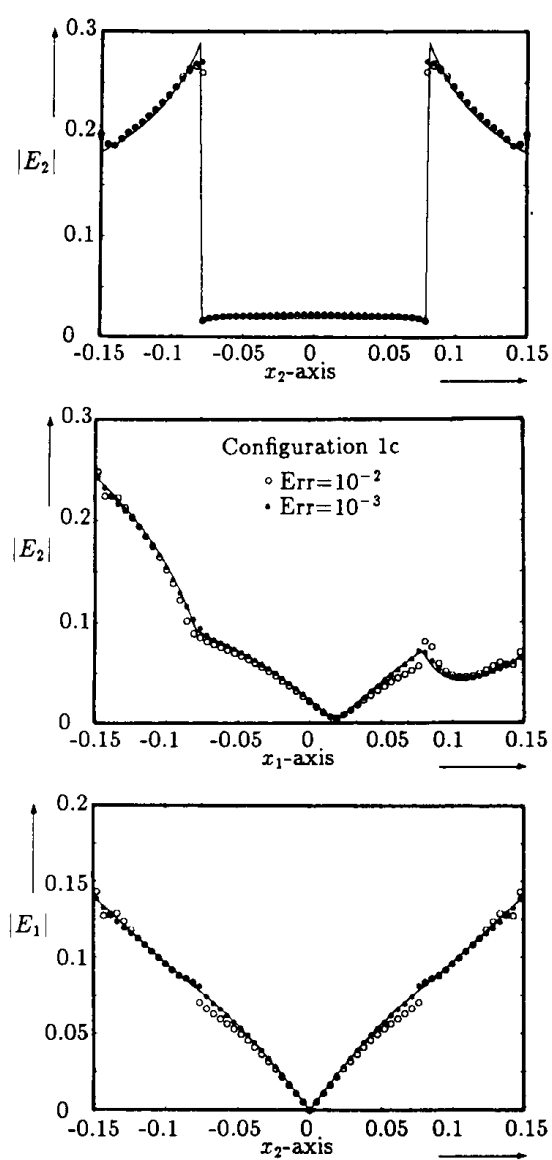

Fig. 4. Comparison between the analytic solution and the present weak formulation for Err $=0.01$ and $\operatorname{Err}=0.001$, respectively, for the first test case.

with proper values of $N_{S}$ and $M_{S}$, the minimum values of $N_{C}=2 N_{S}$ and $M_{C}=2 M_{S}$ will be sufficient to carry out the convolution of (29), provided $\boldsymbol{x}_{n, m}^{(p)} \in S$. This has been observed by Barkeshli and Volakis [12].

\section{Numerical Results}

The numerical convergence is measured by the normalized root-mean-square error, Err:

$$
\operatorname{Err}=\frac{\left\|\boldsymbol{r}^{(n)}\right\|}{\left\|\boldsymbol{r}^{(0)}\right\|}
$$

in which $\left\|\boldsymbol{r}^{(n)}\right\|$ denotes the norm of the residual error in the satisfaction of the operator equation of (16) and (17) over the domain $S$ of the dielectric object in the $n$th iteration. All computations were carried out on a VAX 3100 workstation. The DFT's are efficiently computed using fast Fourier transform (FFT) algorithms. The iteration process is stopped when the normalized root-meansquare error falls below $10^{-3}$. Demonstrating that this strong error criterion has to be imposed for our tests cases, we also present numerical results for one test case 

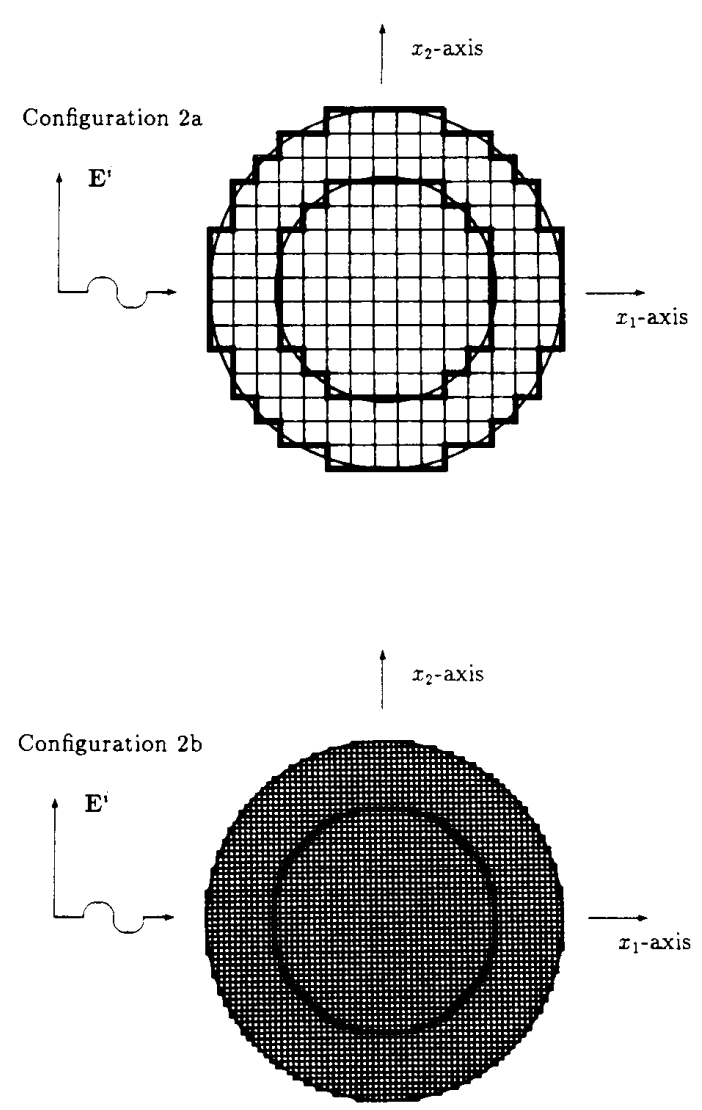

Fig. 5. The different geometries of the coaxially layered cylinder of the second test case.

wherein the iteration process is stopped as soon as the normalized root-mean-square error is less than $10^{-2}$. The incident field is taken to be a uniform plane wave propagating along the $x_{1}$ axis, with the unit electric field vector parallel to the $x_{2}$ axis. In all cases we have taken a zero initial estimate. For every test case presented in this paper the numerical results are compared with the analytical results presented by solid lines in the figures. The origin $x=(0,0)$ is located at the center of the dielectric object; the magnitude of the electric field component $E_{2}$ is shown along the $x_{1}$ and $x_{2}$ axes, while the magnitude of the electric field $E_{1}$ is shown along the $x_{2}$ axis.

In our first test case we take the coaxially layered cylinder where the inner layer (of radius $r=7.9 \mathrm{~cm}$ ) consists of muscle with $\epsilon_{r}=72$ and $\sigma=0.9 \mathrm{~S} / \mathrm{m}$. The outer layer (of radius $r=15 \mathrm{~cm}$ ) consists of fat with $\epsilon_{r}=7.5$ and $\sigma=0.048 \mathrm{~S} / \mathrm{m}$. We consider three different discretizations, shown in Fig. 1. The frequency of the incident field is $100 \mathrm{MHz}$. First we have used a mesh of $N_{S} \times M_{S}=16 \times 16$ (Fig. 1(a)) and $N_{C} \times M_{C}=32 \times 32$. In order to investigate the discretization error, we have subdivided the geometry of Fig. 1(a) to give a mesh size of $N_{S} \times M_{S}=31 \times 31 \quad$ (Fig. $1(\mathrm{~b})$ ) and $N_{C} \times M_{C}=64 \times 64$. Further, we have increased the number of mesh points to
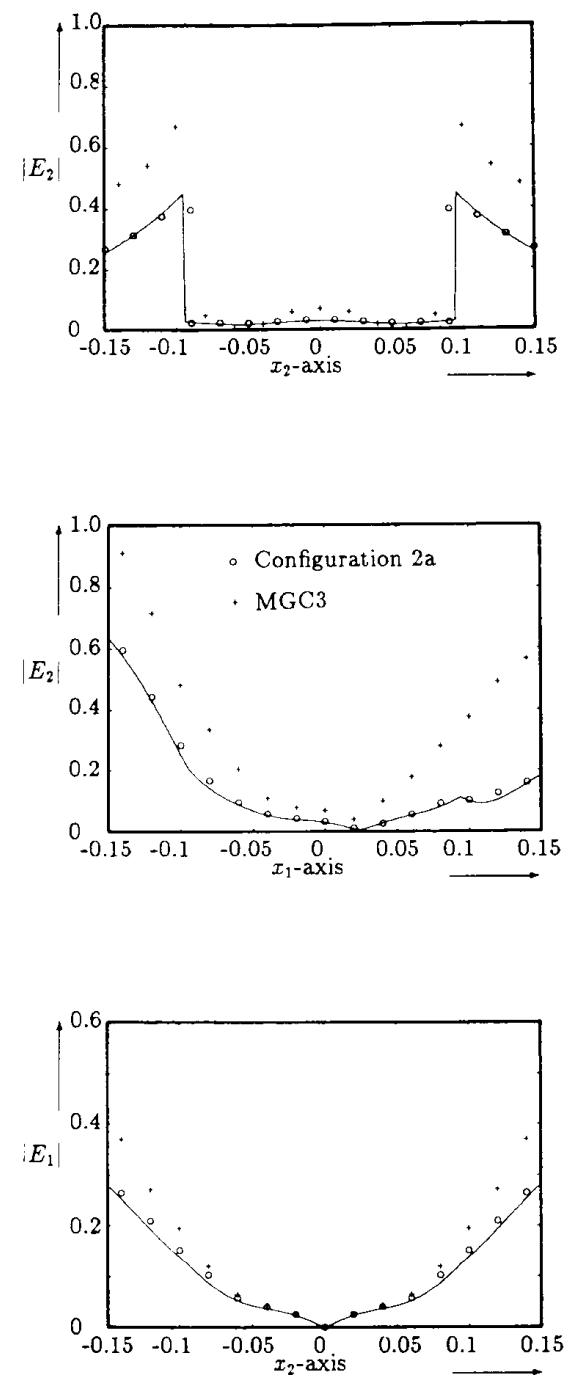

Fig. 6. Comparison between the analytic solution, MGC3 [10], and the present weak formulation for the second test case.

obtain a better approximation of the coaxially layered cylinder using a mesh size of $N_{S} \times M_{S}=64 \times 64$ (Fig. 1(c)) and $N_{C} \times M_{C}=128 \times 128$.

The numerical results associated with these configurations are presented in Figs. 2, 3, and 4. Fig. 2 shows the numerical convergence rate for the geometries of parts (a), (b), and (c) of Fig. 1. It is observed that the present weak formulation reaches the error criterion, Err, of less than $10^{-3}$ within a reasonable number of iterations. Fig. 3 presents the magnitudes of the electric field for the geometries of parts (a) and (b) of Fig. 1 as well as the analytic solution. It is observed that for a mesh of $16 \times 16$ (the geometry of Fig. 1(a)) good numerical results are arrived at. The differences between the analytical results and the numerical results are caused by the staircase 

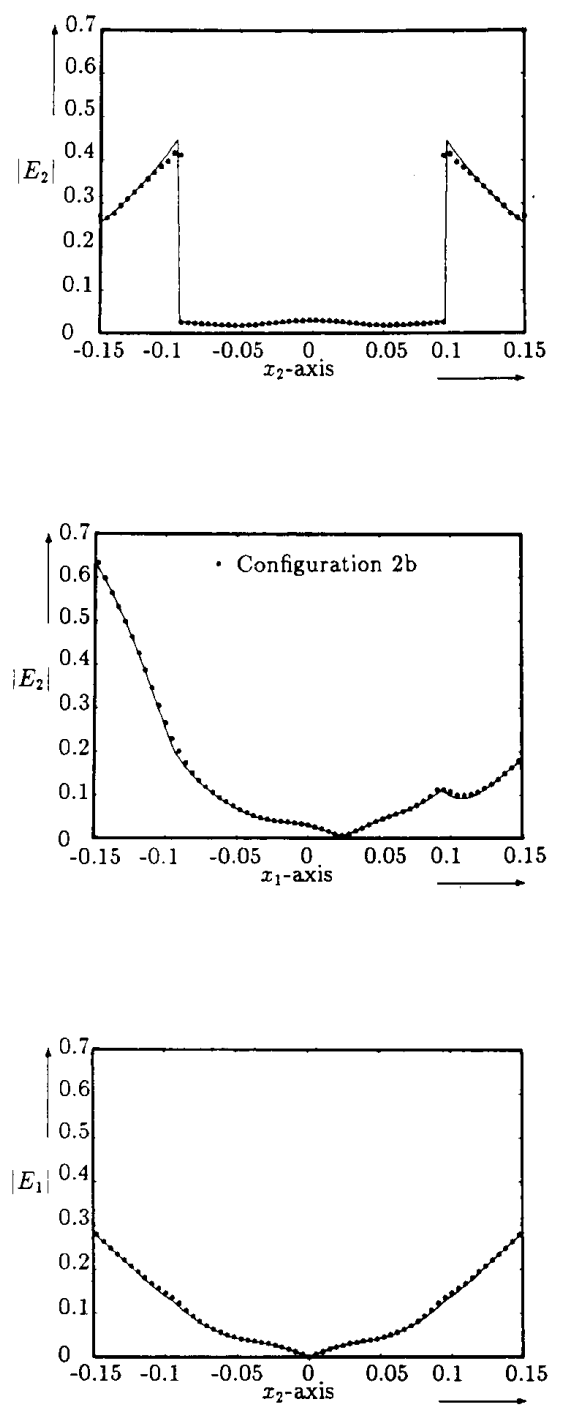

Fig. 7. Comparison between the analytic solution and the present method for the second test case.

approximation of the curved boundary. The numerical results with respect to a more accurate approximation of the circular boundaries (Fig. 1(c)) are shown in Fig. 4, together with the numerical results with error criterions of $1 \%$ and $0.1 \%$, respectively. In Fig. 4 it is demonstrated that an error criterion of $1 \%$ is too weak, while for an error criterion of $0.1 \%$ excellent agreement between the present method with the analytical solutions is arrived at.

In our second test case we take the coaxially layered cylinder where the inner layer (of radius $r=9.4 \mathrm{~cm}$ ) consists of muscle with $\epsilon_{r}=54$ and $\sigma=1.4 \mathrm{~S} / \mathrm{m}$. The outer layer (of radius $r=15 \mathrm{~cm}$ ) consists of fat with $\epsilon_{r}=5.7$ and $\sigma=0.05 \mathrm{~S} / \mathrm{m}$. We consider two different discretizations, shown in Fig. 5. The frequency of the incident field is $300 \mathrm{MHz}$. First we have used a mesh of $N_{S} \times M_{S}=16 \times 16$ (Fig. 5(a)) and $N_{C} \times M_{C}=32 \times 32$ and we have used a mesh of $N_{S} \times M_{S}=64 \times 64$ (Fig. 5(b)) and $N_{C} \times M_{C}=128 \times 128$. The numerical results associated with these geometries are presented in Figs. 6 and 7. The numbers of iterations to obtain an error less than $10^{-3}$ are 224 and 244, respectively. These results are compared with the analytical results of the coaxially layered cylinder. Fig. 6 compares, for the geometry of Fig. 5(a), the magnitudes of the electric field with the numerical results of the MGC3 method (of Joachimowicz and Pichot [10]). It is observed that the present weak formulation performs substantially better than MGC3.

\section{CONCLUSIONS}

We have presented a weak formulation of the conjugate gradient FFT method for dielectric scatterers. It is observed that the present weak form of the conjugate gradient FFT method for 2-D TE scattering problem yields excellent agreement with the analytical results for the test problems. Modeling the curved boundaries using a rectangular mesh seems to be feasible and discretization errors tend to vanish for increasingly finer discretizations.

Since we have maintained the simple convolution structure of the vector potential, we do not have matrix-vector multiplications in the spectral domain. We only have matrix-vector multiplications in the spatial domain, but these are over the domain of the dielectric object only. All told, this means that the computation time of our present method is even less than the computation time of the conjugate gradient FFT methods discussed in the Introduction.

We have solved the integral equation for the unknown electric flux density, $\boldsymbol{D}$; hence the enforced continuity of $D$ yields a correct implementation of the boundary condition at the interfaces of discontinuity. Therefore we have obtained a much simpler scheme than the one of Joachimowicz and Pichot [10]. In the present scheme the surface integrals that are directly related to surface charges at the surfaces of discontinuity do not occur.

Borup et al. [8] have demonstrated that the method of finite differences in the time domain (FD-TD) exhibits better numerical performance than the conjugate gradient FFT methods. Numerical results presented in this paper tend to demonstrate that the present weak form of the conjugate gradient FFT method produces more reliable and efficient results. At this moment it is not clear which method exhibits better efficiency and performance. Therefore, extensive research on both methods seems to be relevant in the future.

\section{REFERENCES}

[1] J. H. Richmond, "Scattering by a dielectric cylinder of arbitrary cross section," IEEE Trans. Antennas Propagat., vol. AP-13, pp. 334-341, May 1965.

[2] J. H. Richmond, "TE wave scattering by a dielectric cylinder of arbitrary cross-section shape," IEEE Trans. Antennas Propagat., vol. AP-14, pp. 460-464, July 1966. 
[3] N. N. Bojarski, " $K$-space formulation of the scattering problem in the time domain," J. Acoust. Soc. Amer., vol. 72, pp. 570-584, 1982.

[4] P. M. van den Berg, "Iterative computational techniques in scattering based upon the integrated square error criterion," IEEE Trans. Antennas Propagat., vol. AP-32, pp. 1063-1071, Oct. 1984

[5] T. K. Sarkar, E. Arvas, and S. M. Rao, "Application of the fast Fourier transform and the conjugate method for efficient solution of electromagnetic scattering from both electrically large and small conducting bodies," Electromagnetics, no. 5, pp. 99-122, 1985.

[6] H. Massoudi, C. H. Durney, and M. F. Iskander, "Limitations of the cubical block model of man in calculating SAR distributions," IEEE Trans. Microwave Theory Tech., vol. MTT-32, pp. 746-752, Aug. 1984.

[7] M. J. Hagmann et al., "Comments on 'Limitations of the cubical block model of man in calculating SAR distributions'" IEEE Trans. Microwave Theory Tech., vol. MTT-33, pp. 347-350, Apr. 1985.

[8] D. T. Borup, D. M. Sullivan, and O. P. Gandhi, "Comparison of the FFT conjugate gradient method and the finite-difference timedomain method for the 2-d absorption problem," IEEE Trans. Microwave Theory Tech., vol. MTT-35, pp. 383-395, Apr. 1987.

[9] A. P. M. Zwamborn and P. M. van den Berg, "A weak form of the conjugate gradient FFT method for plate problems," IEEE Trans. Antennas Propagat., vol. 39, pp. 224-228, Feb. 1991.

[10] N. Joachimowicz and C. Pichot, "Comparison of three formulations for the 2-D TE scattering problem," IEEE Trans. Microwave Theory Tech., vol. 38, pp. 178-185, Feb. 1990.

[11] C. H. Chan and R. Mittra, "Some recent developments in iterative techniques for solving electromagnetic boundary value problems," Radio Sci., vol. 22, pp. 929-934, Nov. 1987.

[12] K. Barkeshli and J. L. Volakis, "On the implementation of the conjugate gradient Fourier transform method for scattering by planar plates," IEEE Antennas Propagat. Magazine, vol. 32, pp. 19-26, Apr. 1990.

[13] E. O. Brigham, The Fast Fourier Transform. Englewood Cliffs, NJ: Prentice-Hall, 1974.

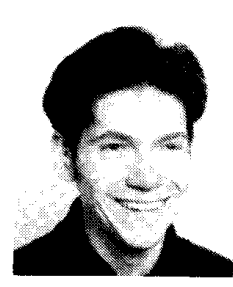

Peter Zwamborn was born in Schiedam, The Netherlands, on October 13, 1963. He received the M.Sc. degree in electrical engineering from the Delft University of Technology in 1987. He is currently working towards the Ph.D. degree in technical sciences at the Delft University of Technology.

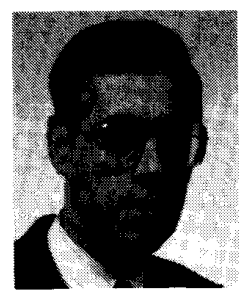

Peter M. van den Berg was born in Rotterdam, The Netherlands, on November 11, 1943. He received the degree in electrical engineering from the Polytechnical School of Rotterdam in 1964. He then received the B.Sc. and M.Sc. degrees in electrical engineering and the Ph.D. degree in technical sciences, all from the Delft University of Technology, in 1966, 1968, and 1971 , respectively.

From 1967 to 1968 , he was employed as a Research Engineer by the Dutch Patent Office. Since 1968, he has been a member of the Scientific Staff of the Electromagnetic Research Group of the Delft University of Technology. During these years, he has carried out research and taught classes in the areas of wave propagation and scattering problems. During the academic year 1973-1974 he was Visiting Lecturer in the Department of Mathematics, University of Dundee, Scotland. During a three-month period in 1980-1981, he was Visiting Scientist at the Institute of Theoretical Physics, Goteborg, Sweden. He was appointed Professor at the Delft University of Technology in 1981. At present, his main research interests are the efficient computation of field problems using iterative techniques based on error minimization and the computation of fields in strongly inhomogeneous media. At the moment he is acting Head of the Laboratory of Electromagnetic Research at the Delft University of Technology. 